\title{
Liebe Leserin, lieber Leser
}

Wer als «Skipper» auf hoher See in Not gerät und Hilfe anfordert, muss gut aufpassen, dass er nicht vom Regen in die Traufe gerät. Lässt er sich in seiner misslichen Lage die Leinen vom Schiff aus zuwerfen, das zu seiner Rettung herbeigeeilt ist, so kann sein eigenes Schiff als «Bergelohn» genommen werden. Er ist dann zwar mit dem Leben davongekommen, sitzt aber ohne Schiff auf dem trockenen. Was hat das mit der «Carepay»-Aktion der Versicherer und der Forderung der Apotheker $\mathrm{zu}$ tun, Rezepte in «Bagatellfällen» selbst ausstellen zu können? Olivier Kappeler, Hochseesegler und Mitglied des Zentralvorstands der FMH, erklärt es Ihnen im Editorial $(\rightarrow$ Seite 573$)$.

Diese Ausgabe der SÄZ hat sich zu einer eigentlichen «Schwerpunktnummer» entwickelt. Gleich drei Themen werden in mehr als einem Artikel behandelt und dadurch von verschiedenen Seiten beleuchtet. In dieser Konzentration ist das zwar ein Zufall, der mit der terminlichen Gebundenheit von zwei der drei Themenschwerpunkte zusammenhängt. Als Zeitschrift, die nicht die Tagesaktualität abdecken kann, möchten wir diese Art der vertieften Auseinandersetzung mit einem Gegenstand aber auch in Zukunft regelmässig pflegen.

Themenschwerpunkt Nr. 1: Per 1. April setzt der Bundesrat das Gesetz über genetische Untersuchungen beim Menschen (GUMG) in Kraft. Zum Gesetz und zu damit zusammenhängenden Verordnungen äussern sich Autoren der Schweizerischen Gesellschaft für medizinische Genetik SGMG, des Bundesamts für Gesundheit BAG sowie des Schweizerischen Versicherungsverbandes SVV $(\rightarrow$ Seite 580, $\rightarrow$ Seite 583 und $\rightarrow$ Seite 586).

Themenschwerpunkt Nr. 2: Über Sinn und Nutzen des Mammographiescreenings wird in den Medien, aber auch in Expertenkreisen und unter Ärzten nach wie vor intensiv diskutiert. Wir haben mit der Schweizerischen Krebsliga sowie dem Dachverband der Schweizer Patientenstellen und der Stiftung für Konsumentenschutz (die Perspektive der beiden letztgenannten Organisationen wird in einem gemeinsamen Artikel wiedergegeben) wichtige Organisationen mit unterschiedlicher Beurteilung der Thematik dazu eingeladen, ihre Sicht der Dinge darzulegen $(\rightarrow$ Seite 594 und $\rightarrow$ Seite 598).

Themenschwerpunkt Nr. 3: «Someone beside you» lautet der Titel eines Dokumentarfilms, in dem sich der Basler Filmemacher und Philosoph Edgar Hagen behutsam, aber mit eindrücklichem Tiefgang dem Phänomen Psychose nähert. Neben der Filmbesprechung finden sich im Kulturteil die Impressionen eines Facharztes sowie ein Gespräch mit dem Regisseur und dem Psychotherapeuten Eric Chapin, dessen Aussagen den Film mitprägen $(\rightarrow$ Seite $617, \rightarrow$ Seite 619 und $\rightarrow$ Seite 623$)$.

Eine Funktion der SÄZ ist es, wichtige Tendenzen im Gesundheitswesen widerzuspiegeln und damit Diskussionen innerhalb der Ärzteschaft, aber auch in weiteren gesundheitspolitischen Kreisen anzuregen. Verschiedene Entwicklungen der letzten Jahre sind für die Ärzteschaft nicht eben erfreulich, was engagierte Kollegen durchaus traurig stimmen kann. Reflexionen über dieses Thema anhand einer Leserzuschrift auf $\Rightarrow$ Seite 626.

Die Redaktion

IMPRESSUM

Redaktion

Dr. med. et lic. phil. Bruno Kesseli

(Chefredaktor)

Daniel Lüthi, FMH

Dr. med. Erhard Taverna

Redaktion Ethik

Dr. theol. Christina Aus der Au

Prof. Dr. med. Lazare Benaroyo

Prof. Dr. phil., dipl. biol.

Christoph Rehmann-Sutter

Redaktion Geschichte

PD Dr. med. et lic. phil. Iris Ritzman

Prof. Dr. med. Jean Jacques Dreifuss

Dr. rer. soc. Eberhard Wolff

Managing Editor

Thomas Heuer, lic. phil

\section{Redaktionssekretariat}

Margrit Neff

Redaktionsadresse

EMH Schweizerischer Ärzteverlag AG Farnsburgerstrasse 8, 4132 Muttenz Tel. 06146785 55, Fax 0614678556 E-Mail: redaktion.saez@emh.ch Internet: www.saez.ch
Herausgeber

FMH

Verbindung der Schweizer Ärztinnen und Ärzte

Fédération des médecins suisses

Federazione dei medici svizzeri

Fwiss Medical Association

Swiss Medical Association

Elfenstrasse 18, 3000 Bern 15

E-Mail: info@fmh.ch

Internet: www.fmh.ch

Verlag

EMH Editores Medicorum Helveticorum

EMH Schweizerischer Ärzteverlag AG

EMH Editions médicales suisses SA

EMH Edizioni mediche svizzere SA

EMH Swiss Medical Publishers Ltd.

Postfach, 4010 Basel

Tel. 06146785 55, Fax 0614678556

E-Mail: verlag@emh.ch

Internet: www.emh.ch

Herstellung

Schwabe AG

Farnsburgerstrasse 8

Postfach 832, 4132 Muttenz

Tel. 06146785 85, Fax 0614678586

E-Mail: druckerei@schwabe.ch
Marketing EMH

EMH Schweizerischer Ärzteverlag AG

Thomas Gierl M.A.

Leiter Marketing und Kommunikation

Farnsburgerstrasse 8, 4132 Muttenz

Tel. 0614678549 , Fax 0614678556

E-Mail: tgierl@emh.ch

Inserate

EMH Schweizerischer Ärzteverlag AG

Ariane Furrer

Assistentin Inserateregi

Farnsburgerstrasse 8, 4132 Muttenz

Tel. 0614678588

Fax 0614678556

E-Mail: afurrer@emh.ch

"Stellenmarkt»

EMH Schweizerischer Ärzteverlag AG

Farnsburgerstrasse 8, 4132 Muttenz

Tel. 06146785 55, Fax 0614678556

E-Mail: stellenmarkt@emh.ch

«Stellenvermittlung»

FMH Consulting Services

Stellenvermittlung

Postfach 246, 6208 Oberkirch

Tel. 04192500 77, Fax 0419210586

E-Mail: fmhstv@hin.ch

Internet: www.fmhjob.ch
Abonnemente

FMH-Mitglieder

FMH Verbindung der Schweizer

Ärztinnen und Ärzte

Elfenstrasse 18, 3000 Bern 15

Tel. 03135911 11, Fax 0313591112

Andere Abonnemente

EMH Schweizerischer Ärzteverlag AG Abonnemente, Postfach, 4010 Basel Tel. 06146785 75, Fax 0614678576 E-Mail: abo@emh.ch

Jahresabonnement: CHF 320.-, zuzüglich Porto

(C) 2007 by EMH Schweizerischer Ärzteverlag AG, Basel. Alle Rechte vorbehalten. Nachdruck, elektronische Wiedergabe und Übersetzung, auch auszugsweise, nur mit schriftlicher Genehmigung des Verlages gestattet.

Erscheint jeden Mittwoch

ISSN 0036-7486

ISSN 1424-4004 (Elektronische Ausg.)
Delegierte der Fachgesellschaften Allergologie und Immunologie: Prof. Dr. A. Bircher

Allgemeinmedizin: Dr. B. Kissling Anästhesiologie und Reanimation Prof. P. Ravussin

Angiologie: Dr. B. Amman-Vesti Arbeitsmedizin: Dr. B. Merz Chirurgie: Prof. Dr. M. Decurtins Dermatologie und Venerologie: PD Dr. S. Lautenschlager Endokrinologie und Diabetologie:

Prof. Dr. G.A. Spinas

Gastroenterologie: Dr. D. Criblez

Geriatrie: Dr. M. Conzelmann

Gynäkologie und Geburtshilfe:
Prof. Dr. Dr. h. c. mult. W. Holzgreve
Hämatologie: Dr. M. Zoppi

Handchirurgie: PD Dr. L. Nagy

Infektologie: Prof. Dr. W. Zimmerli

Innere Medizin: Dr. W. Bauer

Intensivmedizin: Dr. C. Jenni

Kardiologie: Prof. Dr. B. Meier

Kiefer- und Gesichtschirurgie:

Dr. C. Schotland

Kinder- und Jugendpsychiatrie: Dr. R. Hotz

Kinderchirurgie: Dr. M. Bittel

Medizinische Genetik: Prof. Dr. P. Miny

Neonatologie: Prof. Dr. H.-U. Bucher

Nephrologie: Prof. Dr. J.-P. Guignard

Neurochirurgie: Prof. Dr. H. Landolt

Neurochirurgie: Prof. Dr. H. Lando

Neurologie: Prof. Dr. H. Mattle
Neuropädiatrie: Prof. Dr. J. Lütschg

Neuropadiatrie: Prof. Dr. J. Lütschg
Neuroradiologie: Prof. Dr. W. Wichman
Nuklearmedizin: Prof. Dr. J. Mülle Onkologie: PD Dr. B. Pestalozzi Ophthalmologie: Dr. A. Franceschetti ORL, Hals- und Gesichtschirurgie: Prof. Dr. J.-P. Guyot

Orthopädie: Dr. T. Böni

Pädiatrie: Dr. R. Tabin

Pathologie: Prof. Dr. G. Cathomas

Pharmakologie und Toxikologie:

Dr. T. Buclin

Pharmazeutische Medizin: Dr. P. Kleist

Physikalische Medizin und Rehabilitation:

Dr. M. Weber

Dr. M. Weber
Plast.-Rekonstrukt. u. Ästhetische Chirurgie:

Prast.-Rekonstrukt.

Pneumologie: Prof. Dr. E. Russi
Prävention und Gesundheitswesen: Dr. C. Junker

Psychiatrie und Psychotherapie: Dr. G. Ebner

Radiologie: Prof. Dr. B. Marincek Radioonkologie: Prof. Dr. R. H. Greiner Rechtsmedizin: Prof. T. Krompeche Rheumatologie: Prof. Dr. M. Seitz Thorax-, Herz- und Gefässchirurgie: Prof. Dr. T. Carrel

Tropen- und Reisemedizin: PD Dr. C. Hatz Urologie: PD Dr. T. Zellweger 